\title{
Second Hankel determinant for a class defined by modified Mittag-Leffler with generalized polylogarithm functions
}

\author{
M. N. M. Pauzia ${ }^{a}$ M. Darus ${ }^{a, *}$, S. Siregar ${ }^{b}$ \\ a School of Modelling and Data Science (Previously: School of Mathematical Sciences), Faculty of Science and Technology, Universiti \\ Kebangsaan Malaysia, Bangi 43600 Selangor D.E., Malaysia. \\ ${ }^{b}$ Department of Science and Biotechnology, Faculty of Engineering and Life Sciences, Universiti Selangor, Batang Berjuntai, Bestari \\ Jaya 45600, Selangor D.E., Malaysia.
}

\begin{abstract}
In this work, a new generalized derivative operator $\mathfrak{M}_{\alpha, \beta, \lambda}^{\mathrm{m}}$ is introduced. This operator obtained by using convolution (or Hadamard product) between the linear operator of the generalized Mittag-Leffler function in terms of the extensivelyinvestigated Fox-Wright ${ }_{\mathrm{p}} \Psi_{\mathrm{q}}$ function and generalized polylogarithm functions defined by

$$
\mathfrak{M}_{\alpha, \beta, \lambda}^{m} f(z)=\mathfrak{F}_{\alpha, \beta} f(z) * \mathfrak{D}_{\lambda}^{m} f(z)=z+\sum_{n=2}^{\infty} \frac{\Gamma(\beta) n^{m}(n+\lambda-1) !}{\Gamma[\alpha(n-1)+\beta] \lambda !(n-1) !} a_{n} z^{n},
$$

where $\mathrm{m} \in \mathbb{N}_{0}=\{0,1,2,3, \ldots\}$ and $\min \{\operatorname{Re}(\alpha), \operatorname{Re}(\beta)\}>0$. By making use of $\mathfrak{M}_{\alpha, \beta, \lambda}^{\mathrm{m}} \mathrm{f}(z)$, a class of analytic functions is introduced. The sharp upper bound for the nonlinear $\left|a_{2} a_{4}-a_{3}^{2}\right|$ (also called the second Hankel functional) is obtained. Relevant connections of the results presented here with those given in earlier works are also indicated.
\end{abstract}

Keywords: Hankel determinant, modified Mittag-Leffler function, polylogarithms functions.

2010 MSC: 30C45, 30C50.

(C)2018 All rights reserved.

\section{Introduction}

Let $\mathcal{A}$ denotes the family of analytic functions in the open unit disk $\mathbb{U}=\{z \in \mathbb{C}:|z|<1\}$ of the form

$$
f(z)=z+\sum_{n=2}^{\infty} a_{n} z^{n}
$$

A function $\mathrm{f}$ is said to be univalent in the domain $\mathbb{U}$, if it is one-to-one in $\mathbb{U}$. Let $\mathcal{S}$ denote the subclass of $\mathcal{A}$ consisting of functions which are univalent in $\mathbb{U}$.

\footnotetext{
*Corresponding author

Email addresses: nazran@unisel.edu.my (M. N. M. Pauzi), maslina@ukm.edu.my (M. Darus), saibah@unisel.edu.my (S. Siregar)
}

doi: $10.22436 /$ jmcs.018.04.06

Received: 2018-01-26 Revised: 2018-10-30 Accepted: 2018-11-16 
Studies of convolution play an important role in geometric function Theory (GFT). By making use of the Hadamard product (or convolution), several new and interesting subclasses of analytic and univalent functions have been introduced and investigated in the direction of well-known concepts such as the subordination and superordination inequalities, integral mean and partial sums, Hankel determinant and so on.

For functions $\mathrm{f} \in \mathcal{A}$ given by (1.1) and $\mathrm{g} \in \mathcal{A}$ given by $\mathrm{g}(z)=z+\sum_{n=2}^{\infty} \mathrm{b}_{\mathrm{n}} z^{\mathrm{n}}$, we define the Hadamard product (or convolution) of $f$ and $g$ by

$$
(f * g)(z)=z+\sum_{n=2}^{\infty} a_{n} b_{n} z^{n}, \quad z \in \mathbb{U} .
$$

In the present paper we introduced a new generalized derivative operator $\mathfrak{M}_{\alpha, \beta, \lambda}^{\mathrm{m}} \mathrm{f}(z)$ obtained by convolution between between the linear operator of the generalized Mittag-Leffler function in terms of the extensively-investigated Fox-Wright ${ }_{\mathrm{p}} \Psi_{\mathrm{q}}$ function and generalized polylogarithm functions defined as follow.

Definition 1.1 ([20]). For $\mathrm{f} \in \mathcal{A}$, the linear operator of the generalized Mittag-Leffler function in term of the extensively-investigated Fox-Wright ${ }_{\mathrm{p}} \Psi_{\mathrm{q}}$ function is defined by $\mathfrak{F}_{\alpha, \beta} \mathrm{f}(z): \mathcal{A} \rightarrow \mathcal{A}$

$$
\mathfrak{F}_{\alpha, \beta} f(z)=z+\sum_{n=2}^{\infty} \frac{\Gamma(\beta)}{\Gamma[\alpha(n-1)+\beta]} a_{n} z^{n},
$$

where $\min \operatorname{Re}\{(\alpha), \operatorname{Re}(\beta)\}>0 ; z \in \mathbb{U}$.

Definition 1.2 ([4]). For $\mathrm{f} \in \mathcal{A}$, the generalized polylogarithms is defined by $\mathfrak{D}_{\lambda}^{\mathrm{m}} \mathrm{f}(z): \mathcal{A} \rightarrow \mathcal{A}$,

$$
\mathfrak{D}_{\lambda}^{m} f(z)=z+\sum_{n=2}^{\infty} \frac{n^{m}(n+\lambda-1) !}{\lambda !(n-1) !} a_{n} z^{n}
$$

where $\mathrm{m} \in \mathbb{N}_{0}=\{0,1,2, \ldots\}, z \in \mathbb{U}$. It is clear that the operator $\mathfrak{D}_{\lambda}^{\mathfrak{m}} \mathrm{f}(z)$ included two unknown derivative operators. Note that $\mathfrak{D}_{0}^{\mathrm{m}}=\mathfrak{D}^{\mathrm{m}}$ and $\mathfrak{D}_{\lambda}^{0}=\mathfrak{D}_{\lambda}$ which are Sălăgean and Ruscheweyh derivative operators respectively.

Thus, in term of Hadamard (or convolution) given by (1.2). We defined the following convolution operator:

Definition 1.3. For $f \in \mathcal{A}$, the generalized Mittag-Leffler function in term of the extensively-investigated Fox-Wright ${ }_{\mathrm{p}} \Psi_{\mathrm{q}}$ function $\mathfrak{M}_{\alpha, \beta, \lambda}^{\mathrm{m}} \mathrm{f}(z)$ and the generalized polylogarithm functions $\mathfrak{D}_{\lambda}^{\mathrm{m}} \mathrm{f}(z)$ is defined by $\mathfrak{M}_{\alpha, \beta, \lambda}^{\mathrm{m}} \mathrm{f}(z): \mathcal{A} \rightarrow \mathcal{A}$,

$$
\mathfrak{M}_{\alpha, \beta, \lambda}^{\mathrm{m}} \mathrm{f}(z)=\mathfrak{F}_{\alpha, \beta} f(z) * \mathfrak{D}_{\lambda}^{\mathrm{m}} \mathrm{f}(z)=z+\sum_{n=2}^{\infty} \frac{\Gamma(\beta) \mathfrak{n}^{\mathrm{m}}(\mathrm{n}+\lambda-1) !}{\Gamma[\alpha(\mathrm{n}-1)+\beta] \lambda !(\mathrm{n}-1) !} \mathrm{a}_{\mathrm{n}} z^{\mathrm{n}},
$$

where $m \in \mathbb{N}_{0}=\{0,1,2, \ldots\}$, and $\min \operatorname{Re}\{(\alpha), \operatorname{Re}(\beta)\}>0 ; z \in \mathbb{U}$.

In [16], Noonan and Thomas defined the $q^{\text {th }}$ Hankel determinant of $f q \geqslant 1$ and $n \geqslant 1$ as

$$
H_{q}(n)=\left|\begin{array}{cccc}
a_{n} & a_{n+1} & \ldots & a_{n+q+1} \\
a_{n+1} & a_{n+2} & \ldots & a_{n+q+2} \\
\vdots & \vdots & \vdots & \vdots \\
a_{n+q-1} & a_{n+q} & \ldots & a_{n+2 q-2}
\end{array}\right| .
$$

Cantor [6] shows the application of Hankel determinant in showing that a function of bounded characteristic in $\mathbb{U}$, i.e., a function which a ratio of two bounded analytic functions with its Laurent series 
around the origin having integral coefficients, is rational. Wilson [21] studied the application of Hankel determinant in meromorphic functions. Noor in [17] have determined the rate of growth of it with bounded boundary and also studied the Hankel determinant for Bazilevic functions in [18]. The Henkel determinant of exponential polynomials were studied by Ehrenborg [7], and Layman in [13] discussed some of its properties.

It is easily observe that for $q=2$ and $n=1$, we will have a classical theorem of Fekete and Szegö [8]

$$
\mathrm{H}_{2}(1)=\left|\begin{array}{ll}
\mathrm{a}_{1} & \mathrm{a}_{2} \\
\mathrm{a}_{2} & \mathrm{a}_{3}
\end{array}\right|
$$

They made an early study for the estimates of $\left|a_{3}-\mu a_{2}^{2}\right|$ when $a_{1}=1$ and $\mu$ real. The well-known result due to them states that if $f \in \mathcal{S}$, then

$$
\left|a_{3}-\mu a_{2}^{2}\right| \leqslant \begin{cases}4 \mu-3, & \text { if } \mu \geqslant 1, \\ 1+2 e^{\left(\frac{-2 \mu}{1-\mu}\right)}, & \text { if } 0 \leqslant \mu \leqslant 1, \\ 3-4 \mu, & \text { if } \mu \leqslant 0 .\end{cases}
$$

In 1969, Keogh and Merkes [12] discussed the sharp estimates for $\left|a_{3}-\mu a_{2}^{2}\right|$ when $f$ is close-to-convex and starlike in $\mathbb{U}$.

For our discussion in this paper, we consider the Hankel determinant of $f \in \mathcal{S}$ for $q=2$ and $n=2$, given by

$$
\mathrm{H}_{2}(2)=\left|\begin{array}{ll}
\mathrm{a}_{2} & \mathrm{a}_{3} \\
\mathrm{a}_{3} & \mathrm{a}_{4}
\end{array}\right|=\mathrm{a}_{2} \mathrm{a}_{4}-\mathrm{a}_{3}^{2},
$$

known as the second Hankel determinant. The determinant $\mathrm{H}_{2}(2)$ has been investigated by several authors. For example, Janteng et al. [10] have studied the sharp bound for the function $\mathrm{f}$ in (1.1), consisting the functions which derivative has a positive real part and have the result $\left|a_{2} a_{4}-a_{3}^{2}\right| \leqslant 4 / 9$. The same author [11] obtained the result for the sharp upper bounds for starlike and convex functions as $\left|a_{2} a_{4}-a_{3}^{2}\right| \leqslant 1$ and $\left|a_{2} a_{4}-a_{3}^{2}\right| \leqslant 1 / 8$, respectively.

The subclass $\mathfrak{M}_{\alpha, \beta, \lambda}^{\mathrm{m}} \mathrm{f}(z)$ is defined as the following.

Definition 1.4. Let $f$ be given by (1.1). Then $f$ is said to be the class $\mathfrak{M}_{\alpha, \beta, \lambda}^{m} f(z)$ if it is satisfies the inequality

$$
\operatorname{Re}\left\{\left[\mathfrak{M}_{\alpha, \beta, \lambda}^{\mathrm{m}} \mathrm{f}(z)\right]^{\prime}\right\}>0, \quad(z \in \mathbb{U}),
$$

where $\mathfrak{M}_{\alpha, \beta, \lambda}^{\mathrm{m}} f(z)$ is a new generalized operator from convolution between the linear operator of the generalized Mittag-Leffler function in term of the extensively-investigated Fox-Wright ${ }_{p} \Psi_{q}$ function introduced by Srivastava et. al [20] and the generalized polylogarithm functions $\mathfrak{D}_{\lambda}^{\mathfrak{m}} f(z)$ introduced by Al-Saqsi and Darus [4].

Various authors studied and investigated the second Hankel determinant for a certain class of analytic functions such as Al-Refai and Darus [3], Abubaker and Darus [1], Al-Abbadi and Darus [2], and Bansal [5].

Motivated by the results obtained by different authors in this direction mentioned above, in the present paper we introduced a new generalized derivative operator $\mathfrak{M}_{\alpha, \beta, \lambda}^{m} f(z)$ and investigate the upper bound for functional $\left|a_{2} a_{4}-a_{3}^{2}\right|$ for the function $f$ belonging to the class $\mathfrak{M}_{\alpha, \beta, \lambda}^{m} f(z)$.

We first state some preliminary lemmas required for proving our results.

\section{Preliminary Results}

Let $\mathcal{P}$ be the family of all functions $p$ analytic in $\mathbb{U}$ for which $\operatorname{Re}\{p(z)\}>0$ and

$$
\mathrm{p}(z)=1+\mathrm{c}_{1} z+\mathrm{c}_{2} z^{2}+\cdots
$$

for $z \in \mathbb{U}$. 
Lemma 2.1 ([19]). If $\mathrm{p} \in \mathcal{P}$, then $\left|c_{k}\right| \leqslant 2$ for each $k$.

Lemma 2.2 ([9]). The power series for $p(z)$ given in (2.1) converges in $\mathbb{U}$ to a function in $\mathcal{P}$ if and only if the Toeplitz determinants

$$
D_{n}=\left|\begin{array}{ccccc}
2 & c_{1} & c_{2} & \ldots & c_{n} \\
c_{-1} & 2 & c_{1} & \ldots & c_{n-1} \\
\vdots & \vdots & \vdots & \vdots & \vdots \\
c_{-n} & c_{-n+1} & c_{-n+2} & \ldots & 2
\end{array}\right|, \quad n=1,2,3, \ldots
$$

and $c_{k}=\bar{c}_{k}$, are all nonnegative. They are strictly positive except for $p(z)=\sum_{k=1}^{l} \rho_{k} p_{0}\left(e^{i t_{k}} z\right), \rho_{k}>0, t_{k}$ real and $t_{k} \neq t_{j}$ for $k \neq j$; in this case $D_{n}>0$ for $n<l-1$ and $D_{n}=0$ for $n \geqslant l$.

This necessary and sufficient condition is due to Carathéodory and Toeplitz and can be found in [9].

Referring to the method shown by Libera and Zlotkiewicz [14, 15], we may assume without restriction that $c_{1}>0$ and rewriting Lemma 2.2 for the cases $n=2$ and $n=3$.

$$
\mathrm{D}_{2}=\left|\begin{array}{ccc}
2 & \mathrm{c}_{1} & \mathrm{c}_{2} \\
\mathrm{c}_{1} & 2 & \mathrm{c}_{1} \\
\overline{\mathrm{c}_{2}} & \mathrm{c}_{1} & 2
\end{array}\right|=8+2 \operatorname{Re}\left\{\mathrm{c}_{1}^{2} \mathrm{c}_{2}\right\}-2\left|\mathrm{c}_{2}\right|-4 \mathrm{c}_{1}^{2} \geqslant 0,
$$

which is equivalent to

$$
2 c_{2}=c_{1}^{2}+x\left(4-c_{1}^{2}\right)
$$

for some $x,|x| \leqslant 1$. Then $D_{3} \geqslant 0$ is equivalent to

$$
\left|\left(4 c_{3}-4 c_{1} c_{2}+c_{1}^{3}\right)\left(4-c_{1}^{2}\right)+c_{1}\left(2 c_{2}-c_{1}^{2}\right)^{2}\right| \leqslant 2\left(4-c_{1}^{2}\right)^{2}-2\left|2 c_{2}-c_{1}^{2}\right|^{2},
$$

and this, with (2.2), provides the relation

$$
4 c_{3}=c_{1}^{3}+2\left(4-c_{1}^{2}\right) c_{1} x-c 1\left(4-c_{1}^{2}\right) x^{2}+2\left(4-c_{1}^{2}\right)\left(1-|x|^{2}\right) z
$$

for some value of $z,|z| \leqslant 1$.

\section{Main Result}

Our main result is the following.

Theorem 3.1. Let the function $\mathrm{f}$ given by (1.1) be in the class $\mathfrak{M}_{\alpha, \beta, \lambda}^{\mathrm{m}} \mathrm{f}(z)$. Then

$$
\left|a_{2} a_{4}-a_{3}^{2}\right| \leqslant \frac{16[\Gamma(2 \alpha+\beta)]^{2}}{9^{m+1}(\lambda+1)^{2}(\lambda+2)^{2}[\Gamma(\beta)]^{2}} .
$$

The result obtained is sharp.

Proof. Since $f \in \mathfrak{M}_{\alpha, \beta, \lambda}^{m} f(z)$, by virtue of Definition 1.4 there exists an analytic function $p \in \mathcal{P}$ in the unit disk $\mathbb{U}$ with $p(0)=1$ and $[\operatorname{Re} p(z)]>0$ such that

$$
\left[\mathfrak{M}_{\alpha, \beta, \lambda}^{\mathrm{m}} \mathrm{f}(z)\right]^{\prime}=p(z) .
$$

Replacing $\left[\mathfrak{M}_{\alpha, \beta, \lambda}^{m} f(z)\right]^{\prime}$ and $p(z)$ with their equivalent series expressions in (3.1), we have for some $z \in \mathbb{U}$.

$$
1+\sum_{n=2}^{\infty} \frac{\Gamma(\beta) n^{m+1}(n+\lambda-1) !}{\Gamma[\alpha(n-1)+\beta] \lambda !(n-1) !} a_{n} z^{n-1}=1+c_{1} z+c_{2} z^{2}+c_{3} z^{3}+\cdots
$$


Upon simplification, we have

$$
\begin{aligned}
1+ & 2 \frac{2^{m}(\lambda+1) \Gamma(\beta)}{\Gamma[\alpha+\beta]} a_{2} z+\frac{3}{2} \frac{3^{m}(\lambda+2)(\lambda+1) \Gamma(\beta)}{2 \Gamma[2 \alpha+\beta]} a_{3} z^{2} \\
& +\frac{2}{3} \frac{4^{m}(\lambda+3)(\lambda+2)(\lambda+1) \Gamma(\beta)}{\Gamma[3 \alpha+\beta]} a_{4} z^{3}+\cdots=1+c_{1} z+c_{2} z^{2}+c_{3} z^{3}+\cdots .
\end{aligned}
$$

Equating coefficients in (3.2) of the like powers $z^{0}, z$, and $z^{2}$, respectively, yields

$$
\left\{\begin{array}{l}
a_{2}=\frac{1}{2} \frac{c_{1} \Gamma(\alpha+\beta)}{2^{m}(\lambda+1) \Gamma(\beta)}, \\
a_{3}=\frac{2}{3} \frac{c_{2} \Gamma(2 \alpha+\beta)}{3^{m}(\lambda+2)(\lambda+1) \Gamma(\beta)} \\
a_{4}=\frac{3}{2} \frac{c_{3} \Gamma(3 \alpha+\beta)}{4^{m}(\lambda+3)(\lambda+2)(\lambda+1) \Gamma(\beta)}
\end{array}\right.
$$

Substituting the values of $a_{2}, a_{3}$, and $a_{4}$ from (3.3) in the second Hankel functional $\left|a_{2} a_{4}-a_{3}^{2}\right|$, it can be easily established that

$$
\left|a_{2} a_{4}-a_{3}^{2}\right|=\frac{1}{(\lambda+1)^{2}(\lambda+2)}\left|\frac{3}{4} \frac{c_{1} c_{3} \Gamma(\alpha+\beta) \Gamma(3 \alpha+\beta)}{8^{m}(\lambda+3)[\Gamma(\beta)]^{2}}-\frac{4}{9} \frac{c_{2}^{2}[\Gamma(2 \alpha+\beta)]^{2}}{9^{m}(\lambda+2)[\Gamma(\beta)]^{2}}\right| .
$$

We make use of Lemma 2.2 to obtain the proper bound on

$$
\frac{1}{(\lambda+1)^{2}(\lambda+2)}\left|\frac{3}{4} \frac{c_{1} c_{3} \Gamma(\alpha+\beta) \Gamma(3 \alpha+\beta)}{8^{m}(\lambda+3)[\Gamma(\beta)]^{2}}-\frac{4}{9} \frac{c_{2}^{2}[\Gamma(2 \alpha+\beta)]^{2}}{9^{m}(\lambda+2)[\Gamma(\beta)]^{2}}\right| .
$$

Now, to simplify our calculation, we let $r=\Gamma(\beta), s=\Gamma(\alpha+\beta), t=\Gamma(2 \alpha+\beta), u=\Gamma(3 \alpha+\beta), v=8^{n}$, and $w=9^{n}$. Thus, equation (3.4) can be written as

$$
\frac{1}{(\lambda+1)^{2}(\lambda+2)}\left|\frac{3}{4} \frac{c_{1} c_{3} s u}{v(\lambda+3) r^{2}}-\frac{4}{9} \frac{c_{2}^{2} t^{2}}{w(\lambda+2) r^{2}}\right| .
$$

By substituting the values of $c_{2}$ and $c_{3}$ from (2.2) along with (2.3) from Lemma 2.2 in (3.4), we get

$$
\begin{aligned}
\frac{1}{(\lambda+1)^{2}(\lambda+2)}\left|\frac{3}{4} \frac{c_{1} c_{3} s u}{v(\lambda+3) r^{2}}-\frac{4}{9} \frac{c_{2}^{2} t^{2}}{w(\lambda+2) r^{2}}\right| \\
=\frac{1}{(\lambda+1)^{2}(\lambda+2)} \mid \frac{\left[27 \operatorname{suw}(\lambda+2)-16 t^{2} v(\lambda+3)\right] c^{2}-64 v(\lambda+3) t^{2}}{144 v w(\lambda+3)(\lambda+2) r^{2}} \\
\quad+\frac{\left[27 \operatorname{suw}(\lambda+2)-16 t^{2} v(\lambda+3)\right]}{72 v w(\lambda+3)(\lambda+2) r^{2}} c^{2}\left(4-c^{2}\right) x \\
\quad-\left(4-c^{2}\right) x^{2} \frac{\left[27 \operatorname{suw}(\lambda+2)-16 t^{2} v(\lambda+3)\right] c^{2}-64 v(\lambda+3) t^{2}}{144 v w(\lambda+3)(\lambda+2) r^{2}}+\frac{3 \operatorname{csu}\left(4-c^{2}\right)\left(1-|x|^{2}\right) z}{8 v r^{2}(\lambda+3)} \mid .
\end{aligned}
$$

By using the facts $|z|<1$ and triangle inequality with taking $c_{1}=c$ and $c \in[0,2]$ shows that

$$
\begin{aligned}
& \frac{1}{(\lambda+1)^{2}(\lambda+2)}\left|\frac{3}{4} \frac{c_{1} c_{3} s u}{v(\lambda+3) r^{2}}-\frac{4}{9} \frac{c_{2}^{2} t^{2}}{w(\lambda+2) r^{2}}\right| \\
& \leqslant \frac{1}{(\lambda+1)^{2}(\lambda+2)}\left\{\frac{\left|27 s u w(\lambda+2)-16 t^{2} v(\lambda+3)\right| c^{4}}{144 v w(\lambda+3)(\lambda+2) r^{2}}+\frac{3 c s u\left(4-c^{2}\right)}{8 v r^{2}(\lambda+3)}\right. \\
& \quad+c^{2}\left(4-c^{2}\right) \rho \frac{\left|27 \operatorname{suw}(\lambda+2)-16 t^{2} v(\lambda+3)\right|}{72 v w(\lambda+3)(\lambda+2) r^{2}}
\end{aligned}
$$




$$
\left.+\left(4-c^{2}\right)(c-2) \rho^{2}\left[\frac{27 \operatorname{suw}(\lambda+2) c-16 t^{2} v(\lambda+3)(c+2)}{144 v w(\lambda+3)(\lambda+2) r^{2}}\right]\right\}=F(c, \rho) \quad \text { for } \quad 0 \leqslant \rho=|x| \leqslant 1 .
$$

We assume that the upper bound for (3.5) attains at the interior point of $\rho \in[0,1]$ and $c \in[0,2]$. Next, we maximize the function $F(c, \rho)$ on the closed square $[0,2] \times[0,1]$. Differentiating $(3.5)$ with respect to $\rho$, we obtain

$$
\begin{aligned}
\frac{\partial F}{\partial \rho}= & c^{2}\left(4-c^{2}\right) \frac{\left|27 \operatorname{suw}(\lambda+2)-16 \mathrm{t}^{2} v(\lambda+3)\right|}{72 v w(\lambda+3)(\lambda+2) \mathrm{r}^{2}} \\
& +\left(4-c^{2}\right)(c-2) \rho\left[\frac{27 \operatorname{suw}(\lambda+2) c-16 \mathrm{t}^{2} v(\lambda+3)(\mathrm{c}+2)}{72 v w(\lambda+3)(\lambda+2) \mathrm{r}^{2}}\right] .
\end{aligned}
$$

From (3.6) we observe that $\frac{\partial F}{\partial \rho}>0$ for $\rho>0$. Thus (3.6) is an increasing function of $\rho$ and hence it cannot have a maximum in the interior of the closed region $[0,2] \times[0,1]$. Moreover, for fixed $c \in[0,2]$ we have

$$
\max _{0 \leqslant \rho \leqslant 1} F(c, \rho)=F(c, 1)=G(c) .
$$

Therefore, by substituting $\rho=1$ in (3.5), upon simplification we obtain

$$
\begin{aligned}
\mathrm{G}(\mathrm{c})=\mathrm{F}(\mathrm{c}, 1)= & \frac{1}{144 v w \mathrm{r}^{2}(\lambda+1)^{2}(\lambda+2)^{2}(\lambda+3)}\left\{54 \operatorname{suw}(\lambda+2)\left(4-\mathrm{c}^{2}\right) \mathrm{c}\right. \\
& +\left|27 \operatorname{suw}(\lambda+2)-16 \mathrm{t}^{2} v(\lambda+3)\right|\left[\mathrm{c}^{4}+2 \mathrm{c}^{2}\left(4-\mathrm{c}^{2}\right)\right] \\
& \left.+\left(4-\mathrm{c}^{2}\right)(\mathrm{c}-2)\left[27 \operatorname{suw}(\lambda+2) c-16 \mathrm{t}^{2} v(\lambda+3)(\mathrm{c}+2)\right]\right\},
\end{aligned}
$$

then

$$
\begin{aligned}
\mathrm{G}^{\prime}(\mathrm{c})= & \frac{1}{144 v w r^{2}(\lambda+1)^{2}(\lambda+2)^{2}(\lambda+3)}\left\{\left(4-3 c^{2}\right)[54 \operatorname{suw}(\lambda+2)]\right. \\
& +4 c\left(4-c^{2}\right)\left|27 \operatorname{suw}(\lambda+2)-16 \mathrm{t}^{2} v(\lambda+3)\right| \\
& +2 \mathrm{c}(2-\mathrm{c})\left[27 \operatorname{suw}(\lambda+2) c-16 \mathrm{t}^{2} v(\lambda+3)(\mathrm{c}+2)\right] \\
& +2\left(4-\mathrm{c}^{2}\right)\left[\left(27 \operatorname{suw}(\lambda+2)(\mathrm{c}-1)-16 \mathrm{t}^{2} v(\lambda+3) c\right]\right\} .
\end{aligned}
$$

From (3.7), we note that $\mathrm{G}^{\prime}(\mathrm{c}) \leqslant 0$ for every $c \in[0,2]$. Therefore, $\mathrm{G}(\mathrm{c})$ is a decreasing function of $\mathrm{c}$ in the interval $c \in[0,2]$, whose maximum values which occur at points of $G$ must be on the boundary of $c \in[0,2]$. However, $\mathrm{G}(\mathrm{c}) \geqslant \mathrm{G}(2)$ and thus $\mathrm{G}$ has maximum value at $\mathrm{c}=0$.

The upper bound for (3.5) corresponds to $\rho=1$ and $c=0$, in which case

$$
\left|a_{2} a_{4}-a_{3}^{2}\right|=\frac{1}{(\lambda+1)^{2}(\lambda+2)}\left|\frac{3}{4} \frac{c_{1} c_{3} s u}{v(\lambda+3) r^{2}}-\frac{4}{9} \frac{c_{2}^{2} t^{2}}{w(\lambda+2) r^{2}}\right|=\frac{16 t^{2}}{9 w r^{2}(\lambda+1)^{2}(\lambda+2)} .
$$

By substituting $r=\Gamma(\beta), t=\Gamma(2 \alpha+\beta)$ and $w=9^{m}$ in (3.8). We have the upper bound

$$
\left|a_{2} a_{4}-a_{3}^{2}\right|=\frac{16[\Gamma(2 \alpha+\beta)]^{2}}{9^{m+1}[\Gamma(\beta)]^{2}(\lambda+1)^{2}(\lambda+2)} .
$$

By setting $c_{1}=0$ and choosing $x=1$ in (2.2) and (2.3), we find that $c_{2}=2$ and $c_{3}=0$. Substituting these values in (3.9), the equality is attained, which shows that our result is sharp.

This concludes the proof of our theorem.

Equality holds for the functions in $\mathfrak{M}_{\alpha, \beta, \lambda}^{\mathrm{m}} \mathrm{f}(z)$ given by

$$
f^{\prime}(z)=\frac{1+z^{2}}{1-z^{2}}
$$

For the choice of $\alpha=1 / 2, \beta=1, \mathrm{~m}=0$, and $\lambda=0$ into Theorem 3.1, we will obtained the result coincides 
with Janteng et al. [10] as shown in the following corollary.

Corollary 3.2. Let $\mathrm{f} \in \mathbb{R}$ given by (1.1) be in the class $\mathfrak{M}_{1 / 2,1,0}^{0} \mathrm{f}(z)$. Then

$$
\left|a_{2} a_{4}-a_{3}^{2}\right| \leqslant \frac{4}{9}
$$

The result obtained is sharp.

\section{Acknowledgment}

The work here is supported by UKM grant: GUP-2017-064. The authors would like to thank the referee for valuable suggestions.

\section{References}

[1] A. Abubaker, M. Darus, Hankel determinant for a class of analytic functions involving a generalized linear differential operator, Int. J. Pure Appl. Math., 69 (2011), 429-435. 1

[2] M. H. Al-Abbadi, M. Darus, Hankel Determinant for certain class of analytic function defined by generalized derivative operator, Tamkang J. Math., 43 (2012), 445-453. 1

[3] O. Al-Refai, M. Darus, Second Hankel determinant for a class of analytic functions Defined by a fractional operator, European Journal of Scientific Research, 28 (2009), 234-241. 1

[4] K. Al Shaqsi, M. Darus, An operator defined by convolution involving the polylogarithms functions, J. Math. Stat., 4 (2008), 46-50. 1.2, 1

[5] D. Bansal, Upper bound of second Hankel determinant for a new class of analytic functions, Appl. Math. Lett., 26 (2013), 103-107. 1

[6] D. G. Cantor, Power series with integral coefficients, Bull. Amer. Math. Soc., 26 (1963), 362-366. 1

[7] R. Ehrenborg, The Hankel determinant of exponential polynomials, Amer. Math. Monthly, 107 (2000), 557-560. 1

[8] M. Fekete, G. Szegö, Eine Bemerkung uber ungerade schlichte funktionen, J. London Math. Soc., 8 (1933), 85-89. 1

[9] U. Grenander, G. Szegö, Toeplitz Forms and their Application, University of California Press, Berkeley-Los Angeles, (1958). 2.2, 2

[10] A. Janteng, S. A. Halim, M. Darus, Coefficient Inequality for a Function whose Derivative has a Positive Real Part, JIPAM. J. Inequal. Pure Appl. Math., 7 (2006), 5 pages. 1, 3

[11] A. Janteng, S. A. Halim, M. Darus, Hankel Determinant for Starlike and Convex Functions, Int. J. Math. Anal. (Ruse), 1 (2007), 619-625. 1

[12] F. R. Keogh, E. P. Merkes, A coefficient inequality for certain classes of analytic functions, Proc. Amer. Math. Soc., 20 (1969), 8-12. 1

[13] J. W. Layman, The Hankel transform and some of its properties, J. Integer Seq., 4 (2001), 11 pages. 1

[14] R. J. Libera, E. J. Zlotkiewicz, Coefficient bounds for the inverse of a function with derivative in $\mathcal{P}$, Proc. Amer. Math. Soc., 87 (1983), 251-289. 2

[15] R. J. Libera, E. J. Zlotkiewicz, Early coefficients of the inverse of a regular convex function, Proc. Amer. Math. Soc., 85 (1982), 225-230. 2

[16] J. W. Noonan, D. K. Thomas, On the second Hankel determinant of areally mean and p-valent function, Trans. Amer. Math. Soc., 223 (1976), 337-346. 1

[17] K. I. Noor, Hankel determinant problem for the class of functions with bounded boundary rotation, Rev. Roumaine Math. Pures Appl., 28 (1983), 731-739. 1

[18] K. I. Noor, S. A. Al-Bany, On Bazilevic functions, Internat. J. Math. Math. Sci., 10 (1987), 79-88. 1

[19] C. H. Pommerenke, Univalent Functions, Vandenhoeck \& Ruprecht, Göttingen, (1975). 2.1

[20] H. M. Srivastava, A. Kilicman, Z. E. Abdulnaby, R. W. Ibrahim, Generalized convolution properties based on the modified Mittag-Leffler function, J. Nonlinear Sci. Appl., 10 (2017), 4284-4294. 1.1, 1

[21] R. Wilson, Determinantal criteria for meromorphic functions, Proc. London Math. Soc., 4 (1954), 357-374. 1 